\title{
Static and dynamic performance of direct hybrid connections of cross-laminated timber with steel, concrete and laminated strand lumber composites
}

\author{
Tulio Carrero $^{a, b^{*}}$ (D) , Jairo Montaño ${ }^{b}$ (D) , Hernán Santa-María ${ }^{a, b}$ (D), Pablo Guindos ${ }^{a, b *}$ (D) \\ aEscuela de Ingeniería, Pontificia Universidad Católica de Chile, Santiago 6904411, Chile. E-mail: tecarrero@uc.cl, hsm@ing.puc.cl, \\ pguindos@ing.puc.cl \\ bCentro de Innovación en Madera CIM UC-CORMA, Pontificia Universidad Católica de Chile, Santiago 6904411, Chile. \\ E-mail: jamontano@uc.cl \\ * Corresponding author
}

http://dx.doi.org/10.1590/1679-78256106

\begin{abstract}
Hybrid multistory buildings are every day more common in the construction industry. However, there is little understanding of the performance of the hybrid connections. In this research, the static and dynamic response of cross-laminated timber (CLT) composites combined with reinforced concrete (RC), hollow stee profiles and laminated strand lumber (LSL) has been investigated. In addition, the effects of posttensioning stresses as well as distinct types of connectors such as nails, self-tapping screws and self-tapping dowels has been accounted for. After experimental work, numerical modelling for simulating the static and dynamic behavior for these hybrid connections was also investigated. Results indicate that such massive timber composite connections behave reasonably similar to conventional timber connections, except in that inelastic deformations may increase up to $200 \%$. In addition, it has been found that existing hysteretic models like the modified Stewart hysteretic model (MSTEW) fit for modelling purposes except that very asymmetric hysteretic behavior can be found for timber-concrete hybrid connections.
\end{abstract}

\section{Keywords}

Hybrid connection, structural connection, composite connection, cross-laminated timber, wood, steel, concrete, laminated strand lumber, cyclic, monotonic.

\section{Graphical Abstract}

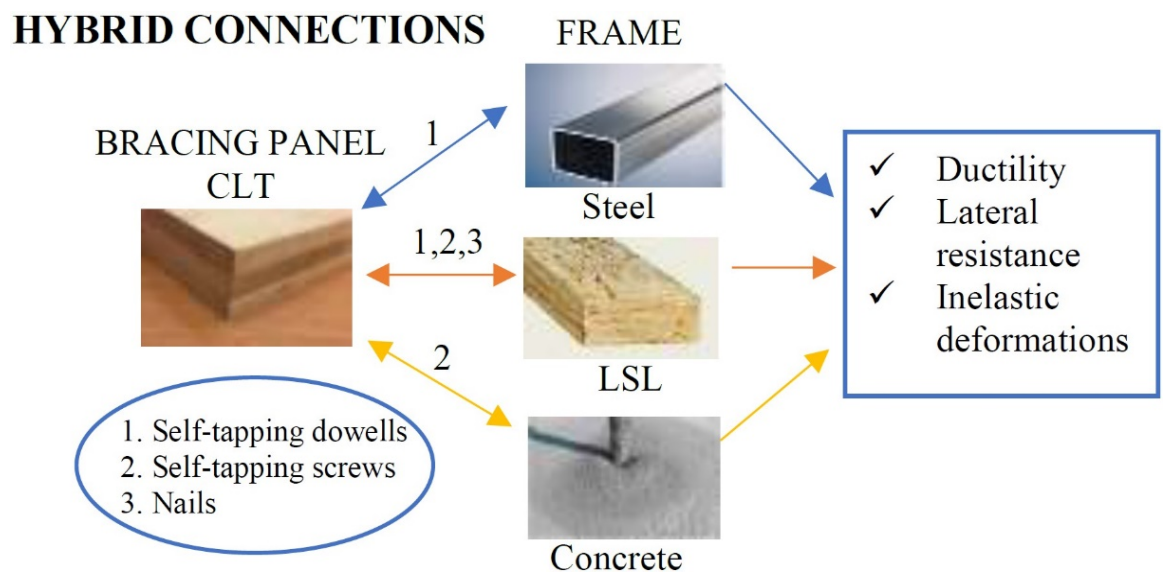




\section{INTRODUCTION}

Cross-laminated timber (CLT) is a laminated composite typically produced by cross gluing 3, 5, 7 or 9 wooden plies with thickness of about $30 \mathrm{~mm}$ each according to a [0/90/0/90]s scheme. This product is commonly produced in the form of panels of about 1,2-3,5 $\mathrm{m}$ in width and 2,4-18 $\mathrm{m}$ in length, casting thus massive boards which are much thicker than customary wooden composite boards such as plywood or oriented strand boards (OSB) (Blaß \& Sandhaas, 2017). Being manufactured by widely spread softwoods like picea Abies or similar species, CLT yields to much stiffer and stronger structures in comparison to conventional wooden constructions. Such enhanced mechanical performance has gained considerable interest in the research community and construction industry because much stiffer and stronger buildings can now be readily constructed (Ringhofer, A. et al., 2018). So far, up to 18-story height CLT buildings have been accomplished (Connolly, T. et al., 2018), while projects and designs for taller buildings have also been reported (Foster, R. M. et al., 2016).

Constructing mid- and especially high-rise timber buildings, still often requires the utilization of hybrid material compositions, which mostly entails the combination of CLT with reinforced concrete (RC), steel or similar structural materials (Foster, R. M. et al., 2016). Even when hybrid structures are of increasing popularity in the global construction industry, material combinations are becoming especially important for CLT buildings in order to enhance lateral stiffness, strength, ductility or energy dissipation among other characteristics (Moore, M., 2000). Among the pioneering medium-height wooden hybrid buildings, the work of Moore in New Zealand is a good example of one of the most sought benefits of early timber hybrid buildings, which consisted of achieving a substantial weight reduction on the slabs of a common concrete building. The lightening of slabs was achieved by composing wooden diaphragms, with structural steel and RC piles. Later, multiple hybrid combinations were proposed for timber buildings taller than six stories (Van De Kuilen et al., 2011). One of the most popular combinations consists of setting the CLT as the wall and/or slab material, while using a structural core made of steel trusses or concrete shear walls, which provides most of the lateral stiffness and strength of the structure (Dunbar A. et al., 2014). Other remarkable applications entail the use of steel or concrete beams with modern CLT slabs/walls (Aicher, S. et al., 2014), whereas other propose hybrid CLT-concrete slabs with concrete thickening as jointing devices (Chapman, J. et al., 2012). In Japan, Koshihara, M. et al., (2013) proposed a concrete shear wall system in the first story, while the second up to the fifth story are made of a hybrid timber-steel bracing system.

In order to use CLT in the aforementioned and future hybrid applications, it is of upmost importance to gather comprehensive understanding of the mechanical behavior at the connections when jointing the different materials (Hashemi, A. et al., 2018). Whether, the different materials are integrated at the component levels (hybrid slab/diaphragms, hybrid beams, hybrid columns, hybrid diagonals, hybrid shear walls) and/or at the building system levels (hybrid shear wall system, tube system, vertical mixed system), the connection behavior must be well understood in order to take the advantages of each material. Such understating gains even more relevance when designing structures under dynamic loads such as wind or earthquakes. The reason being that the connections can highly influence the response of the overall structure, as they are the main responsibles of ensuring deformation compatibility between the two materials (Hashemi, A. et al., 2016). In fact, widely spread international design methods require the definition of monotonic and/or cyclic behavior for these joints not only in elastic, but inelastic range (Federal Emergency Management Agency, 2000, 2009) and (European Committee for Standardization, 2009). However, the mechanical behavior of hybrid connections for CLT structures under static and dynamic loads is rather unknown.

More precisely, there is multiple connection hardware available in the market that has been mainly designed to join common CLT structures, i.e. non hybrid buildings, and eventually can be used as indirect connection devices for jointing hybrid structures as well. The most typical hardware being common steel angle brackets, which are typically used to connect CLT walls to CLT slabs, but they also can be applied to connect CLT walls onto concrete foundations (Blaß \& Sandhaas, 2017). This type of indirect jointing devices has been extensively investigated both experimentally (Schneider, J et al., 2014) and theoretically (Hummel, J., 2017), and therefore it has been used in several hybrid applications. For instance, common steel angle brackets were used to connect CLT as lateral stiffening members of moment resisting steel frames Dickof, C. (2013) and Kanazawa, K. et al. (2018) or energetic retrofit of masonry buildings (Kanazawa, $\mathrm{K}$. et al., 2018). The main reason to use indirect connection technologies being the lack of understanding of using direct joints, as the later are customarily cheaper and faster. In Australia, Hassanieh, A. et al. (2016) tested the use of direct connections with bolts and screws as direct connection method for CLT to steel beams for prefabricating timber flooring systems. The investigation was focused on understanding the static behavior proposing a load-slip model based on non-linear regression of the monotonic curve. In Italy, similar connections were tested in Loss, C. et al. (2016) including experiments reversed cyclic experiments to comprehend the dynamic behavior of direct jointing. The objective of this study was to assess hybrid steel-timber prefab modules as an efficient lightweight structure in seismic areas. From the two previous 
researches, it is concluded that direct jointing is of interest not only for constructing hybrid buildings but specially to achieve more efficient and effective assemblies at prefabrication plants.

Apart from the general scarceness of research in the field of direct jointing, it is important to note that this type of jointing devices may be often subjected to considerable post-tensioning stresses in tall wooden buildings, which eventually might modify the mechanical response of the connections. In fact, the post-tensioning methods are everyday more common to increase lateral stiffness, strength and ductility of walls, columns and beams of timber buildings, which is achieved by using post-tensioned steel tendons according to lqbal, A. et al. (2015) and Wu et al. (2018). Under these circumstances, the mechanical response of the connections can be substantially modified due to increasing compression and thus friction forces at the shear plane of the connection, which is commonly referred as rope effect, as well as modifications in the deformed shape of the connectors as it is observed in reinforced connections (Blaß \& Sandhaas, 2017). Similar behavior has been found in connections of un-tensioned high-rise buildings simply because of large gravitational loads at the first stories. Indeed, recent investigations proofed significant increases in strength, stiffness and energy dissipation of components and the entire structure, which mainly has been attributed to the compression forces and frictions at the connections (Guíñez, F. et al., 2019).

Therefore, this investigation comprises the determination of the monotonic and cyclic behavior of direct hybrid connections when combining CLT with steel, concrete and laminated strand lumber (LSL). Although the latter material has not yet been widely used for the construction of multistory timber buildings, its high homogeneity, large production sizes and strong mechanical properties suggest that it also could fit such purpose (Aburto, V., 2014). This investigation does not only cover distinct material combinations but also direct jointing devices such as nails, self-tapping screws and steel self-tapping dowels. The variability in the connection devices is of interest because not only different fasteners are needed for connecting different materials, but also multiple fasteners can be used for jointing each particular material combination (Hassanieh, A. et al., 2016). This investigation is, however, restricted to mechanical connections because glued and contact joints are not widely used for hybrid timber structures due to considerable brittleness which may be critical under dynamic loading. In addition, posttensioning stresses were also considered for the investigated CLT-concrete connection in order to elucidate the potential influence of axial loading on strength, stiffness and energy dissipation at the connection level. The experimental results of the hybrid connections are compared against results obtained on conventional connections (European Committee for Standardization, 2016a). Furthermore, the numerical modelling of these connections is also accomplished, and the suitability of a monotonic and dynamic model is analyzed.

\section{TEST PROGRAM}

\subsection{Materials and Methods}

Three types of CLT hybrid joints were investigated in this research, including CLT-steel, CLT-LSL and CLT-RC connections, being the latter tested both with (CLT-RCW) and without posttensioning stress (CLT-RCwo). The CLT was produced by gluing Chilean Pinus radiata boards of 20×150 mm in cross section and C24 grading class according to European Committee for Standardization (2016a) at the fabrication plant from Forestal Tricahue Ltda., Coronel, Chile. The gluing was performed at both the edges and faces of the boards casting a 3-ply laminate of $60 \mathrm{~mm}$ in thickness. The CLT density was $476 \mathrm{~kg} / \mathrm{m}^{3}$ in average and moisture content prior was $12 \%$. According to previous studies, the mechanical properties of this Chilean CLT is similar to common European CLT produced with C24 boards (Aburto, V., 2014). The steel components consisted of A36 structural steel hollow section of $100 \times 200 \mathrm{~mm}$ and $6 \mathrm{~mm}$ in thickness. Two types of RC members were investigated. The first RC member measured $330 \times 200 \mathrm{~mm}$ in cross section and used concrete of average compression strength of $38,6 \mathrm{MPa}$ at 28 days, being resistance measured by testing 3 samples according to conventional cylinder compression procedure with the Chilean Standard $\mathrm{NCH} 1037$. The second RC member was similar to the first, except that it was post-tensioned with two threated A36 steel bars of $16 \mathrm{~mm}$ in diameter, which were posttensioned with a force of $54 \mathrm{kN}$ via nut torque or turn-of-nut method $(75.6 \mathrm{kN} . \mathrm{mm})$. The compression force was selected to simulate typical loading of shear wall studs in multistory timber buildings considering axial and overturning effects. The LSL members measured $240 \times 90 \mathrm{~mm}$ in cross-section with an average density of $650 \mathrm{~kg} / \mathrm{m}^{3}$, and grading class $1.75 \mathrm{E}$ according to LP Building Products (2019), and were produced by LP Corporation at the plant of Houlton, ME, USA.

Three different fasteners were considered as the mechanical connection devices. The first was a steel self-tapping dowel WS of $7 \mathrm{~mm}$ in diameter and $233 \mathrm{~mm}$ in length, produced by Rothoblaas Srl., Cortaccia, Italy. These dowels are capable to self-perforating thin steel plates, so they were used in the CLT-steel connection specimen (Figure 1(a)). Also, for comparison purposes, the dowels were also tested in the CLT-LSL connections. Second, a common wood self-taping screw TX30 of $7 \mathrm{~mm}$ in diameter and $320 \mathrm{~mm}$ in length produced by Rothoblaas Srl. was used for both the CLT-RC and 
CLT-LSL connections. Third, an annular ring 40d nail of $5 \mathrm{~mm}$ in diameter and $127 \mathrm{~mm}$ in length produced by Simpson Strong-Tie Company Inc., Pleasanton, CA, U.S.A., was used for the CLT-LSL connection, such that for this hybrid joint both self-tapping screws (CLT-LSLs), nails (CLT-LSLn) and dowels (CLT-LSLd) were investigated, see Figure 1.

(a) CLT-Steel

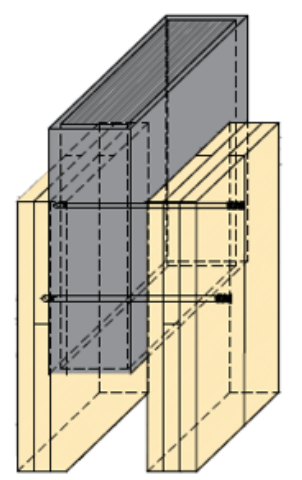

(b) CLT-RCwo

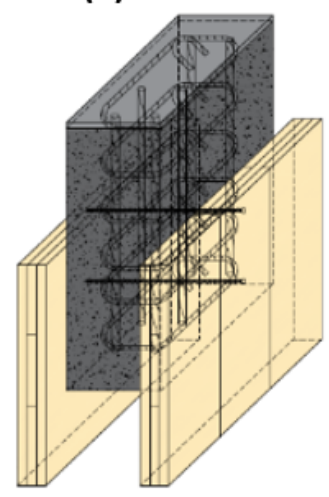

(e) CLT-LSLn

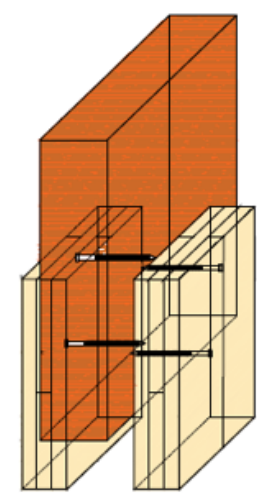

(c) $\mathrm{CLT}-\mathrm{RCW}$

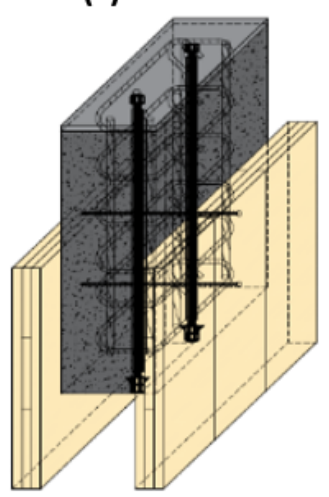

(f) CLT-LSLd
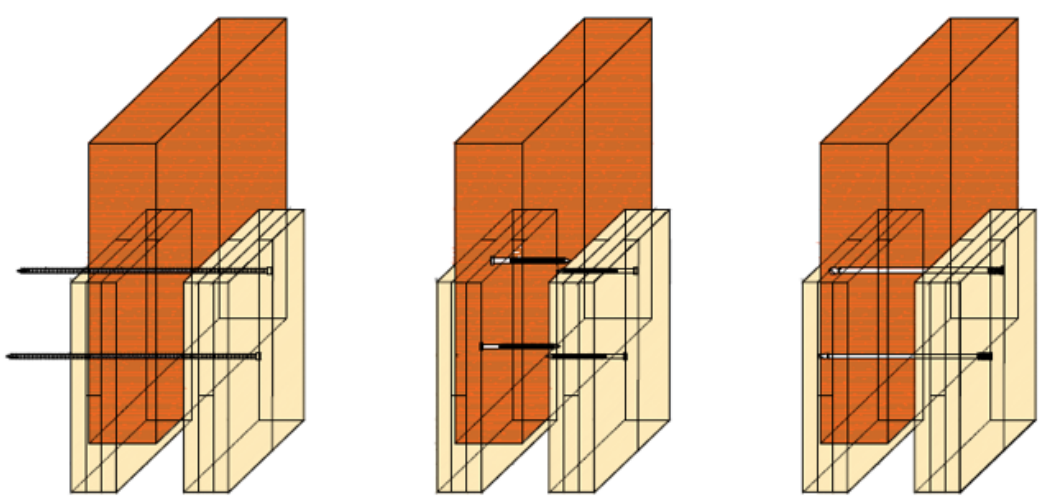

(g) Self-tapping dowels

(h) Self-tapping screws

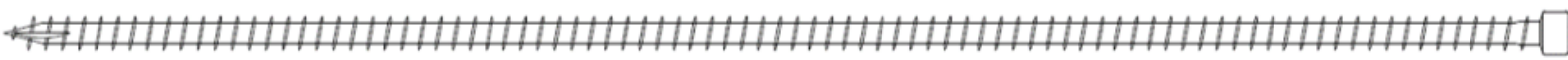

(i) Annular ring nails

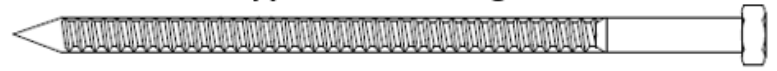

Figure 1. Schematics and labelling of the tested connections and connecting devices including: (a) CLT-Steel connection with self-tapping dowels; (b) CLT-RC connection with self-tapping screws and without posttensioning stress; (c) CLT-RC connection with self-tapping screws and posttensioning stress; (d) CLT-LSL connection with self-tapping screws; (e) CLT-LSL connection with annular ring nails; (f) CLT-LSL joint with dowels; $(\mathrm{g})$ detail of self-tapping dowels; $(\mathrm{h})$ detail of self-tapping screws; (i) detail of annular ring nails.

The design of the connections was accomplished considering the European Yield Model (EYM) of European Committee for Standardization (2016b) by assuming that the CLT-LSL joint could be designed as normal wood-to-wood connection, and both the CLT-steel and CLT-RC joints could be designed as wood-to-thick steel plate connections. More specifically, the CLT-steel connection was designed assuming that the thick plate thickness was twice the hollow-section thickness. In the case of CLT-RC, thick steel plate condition was considered because negligible deformation of fasteners within concrete was assumed in comparison to the stiffness of the wood. The fastening spacing in the CLT was set according to European Committee for Standardization (2005). The minimum required thicknesses of members were verified to ensure ductile failure modes in all connections because this failure mode is preferred. All connections were designed symmetrically to avoid eccentricities and consequent overturning moments during testing. Schematic view of the connections' and labelling are shown in Figure 1 along with the fasteners used.

The testing program consisted of the monotonic and cyclic testing of the connections according to the European wooden connections testing protocol, EN 12512 (European Committee for Standardization, 2005). Except for the CLT-RC 
connections, for which only two cyclic tests were conducted, two monotonic and two cyclic tests were performed for each connection configuration, comprising a total of 22 tests, 10 monotonic and 12 cyclic. According to EN 12512, the monotonic testing must be performed by controlling a displacement rate of less than $0,2 \mathrm{~mm} / \mathrm{s}$ until the ultimate displacement $\left(\delta_{\mathrm{u}}\right)$ is reached, which corresponds to either the connection failure displacement, the displacement when capacity is reduced to $80 \%$ of the maximum measured force, or a maximum displacement of $30 \mathrm{~mm}$. However, we let the actuator to overcome the $30 \mathrm{~mm}$ restraint, because as discussed later, the ultimate displacements of this massive hybrid connections can be much larger in comparison to common connections. Still, the monotonic testing time lapse did not last more than 120 additional seconds in comparison to the conventional testing protocol, hence rate effects still were assumed negligible. The definition of the yielding point, corresponding to the yielding displacement, $\delta_{y}$, and yielding load, $F_{y}$, was established as the intersection between two lines. The first, whose slope determines the connection stiffness, $\mathrm{K}$, corresponds to the secant defined by the $10 \%$ and $40 \%$ force points respect to the maximum load. The second line is the tangent to the monotonic curve, whose slope is $1 / 6$ of the first line, see graphical description in the Figure 2(a). Figure 2 shows experimental protocol and parameters' identification protocol according to EN 12512 including: (a) identification of parameters from the monotonic curve; (b) cyclic protocol scaling according to yielding displacement; (c) determination of strength degradation according to the difference of the hysteretic capacities between the first and third repetition; the results of the monotonic curve allow for defining the connection ductility as $D=\delta_{u} / \delta_{y}$.

(a)

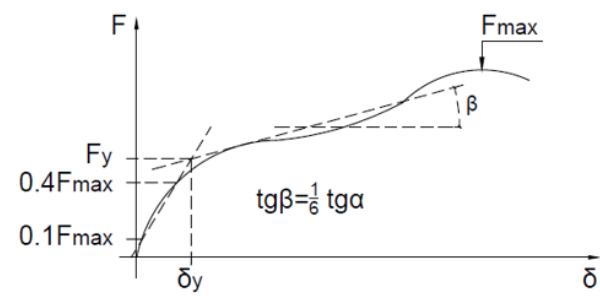

(b)

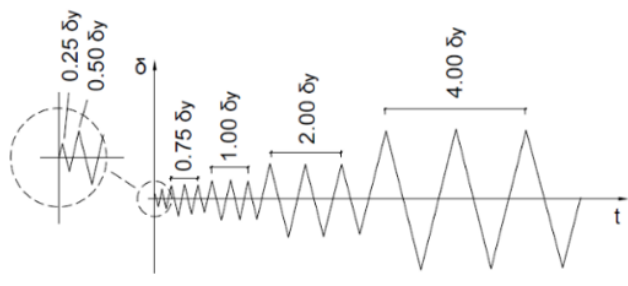

(c)

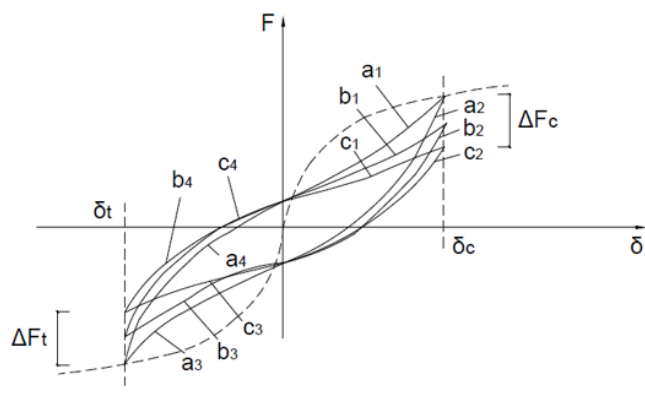

Figure 2. Experimental protocol and parameters' identification protocol according to EN 12512 including: (a) identification of parameters from the monotonic curve; (b) cyclic protocol scaling according to yielding displacement; (c) determination of strength degradation according to the difference of the hysteretic capacities between the first and third repetition.

The cyclic protocol was performed as stated in EN 12512, such that three repetitions of each displacement amplitude were applied, while displacement amplitude was increasingly scaled according to monotonic yielding displacement, see Figure 2(b). According to the resulting hysteresis curve, the EN 12512 states that strength degradation corresponds to the absolute value of capacity drop, $\Delta \mathrm{F}$, between the first and third repetition both for tension and compression (Figure 2(c)). The energy dissipation, $E_{d}$, is calculated as the area within the hysteresis curve, while the 
equivalent viscous damping can be obtained by equivalent linearization considering the potential energy $\left(E_{p}\right)$ and $E_{d}$ such that:

$v_{e q}=E_{d}\left(2 \pi E_{p}\right)$

In addition to the EN 12512 standard parameter identification protocol, the stiffness degradation, $\Delta \mathrm{K}$, shown in the hysteresis curve was also determined. The quantification was analogous to the strength degradation, such that $\Delta K$ was given by the slope difference between the stiffness secants of the first and third repetition, both for tension and compression. The relative displacement at the shear planes was recorded by averaging the measurements of two LVDTs whose displacement was constrained by small steel brackets located at the central member near to each shear plane. In order to hold down the connections during tension cycles, a tension accessory consisting of bolts and steel profiles was holding each side member. The testing was performed at an universal testing machine UE34300 UTM, Laryee Technology Co.,Ltd., Beijing, China. The detail of the testing layout and tension accessories is shown in Figure 3.

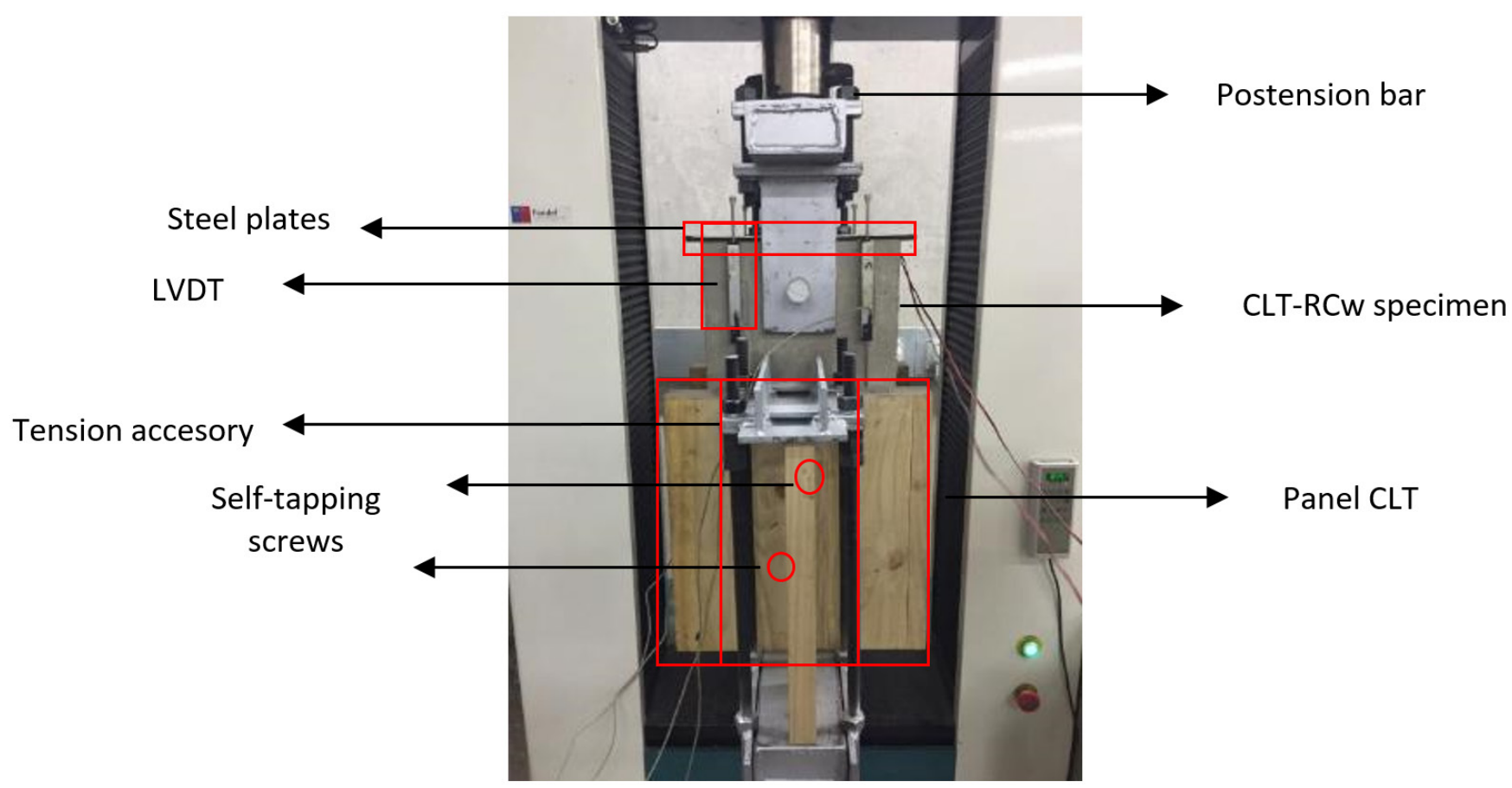

Figure 3. Mechanical testing of the connections according to EN 12512 including the layout for the CLT-RCwo connection along with the tension accessories to hold down the side members during cyclic testing.

\section{TEST RESULTS AND DISCUSSION}

\subsection{Monotonic tests}

The measured load-slip curves of the CLT-Steel and CLT-LSL monotonic tests are shown in Figure 4. The CLT-RC connections were tested only under reversed cyclic load in order to determine the potential effects of friction with and without posttensioning forces. The load-slip curves presented in Figure 4 are the average of two monotonic tests, and the load is the force per fastener and shear plane as defined in European Committee for Standardization (2016b). For comparison purposes, a typical monotonic response of conventional nails in wood to wood connections $(42 \times 185 \mathrm{~mm}$ beams from pine) obtained in General Building Inspectorate Approval (2014) are also shown in the Figure 4. A summary of the measured monotonic parameters of the hybrid connections is presented in Table 1. The cyclic protocol and standard parameter identification protocol used in the monotonic test was performed as stated in EN 12512. 


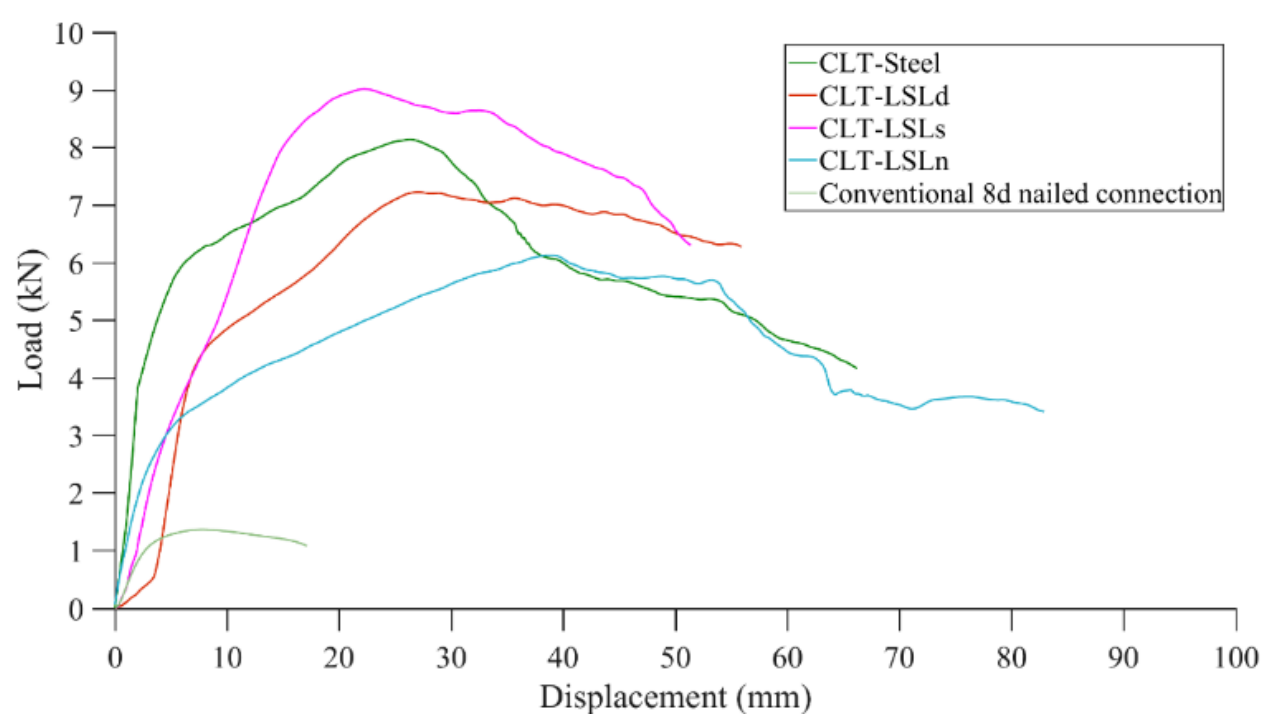

Figure 4. Monotonic load-slip curves of the hybrid connections and comparison with a conventional $8 \mathrm{~d}$ nailed connection. The results shown correspond to the capacity per fastener and shear plane.

One of the most remarkable results illustrated in Figure 4 is that all the fasteners used in the CLT hybrid connections provided not only higher strength in comparison to conventional timber connections but also much larger inelastic deformations. Maximum displacements of conventional wooden connections are commonly smaller than $30 \mathrm{~mm}$, which is the main reason for defining the maximum displacemente in the test to $30 \mathrm{~mm}$ and setting the actuator permisible velocities in European Committee for Standardization (2005). For the tested connections however, the maximum displacements were as large as $50-80 \mathrm{~mm}$. Such larger inelastic displacements are atributed to the fact that the connections were designed for ductile failing modes, in which the maximum displacement is mainy given by the ultimate curvature of the fastener such that the longer fasteners required in the tested hybrid CLT connections allow for providing much larger yielding displacements under similar ultimate curvatures. In this case, the self-reinforcing effect of the transverse layers of the CLT reported by other authors (European Cooperation in Science \& Technology, 2018) is assumed not to greatly influence in enlarging maximum displacements because no brittle failures were observed in the CLT layers located at the shear plane of the connections. Although predictable and highly ductile behavior was found for most of the tested connections, brittle behavior was observed for the CLT-RC connections. The relevant failure modes observed in the distinct connections are presented in Figure 5 to 8. Especially for the post-tensioned concrete connections, abrupt failure loading at premature loading stages can be expected due to brittle shear failure of the concrete at the shear plane.
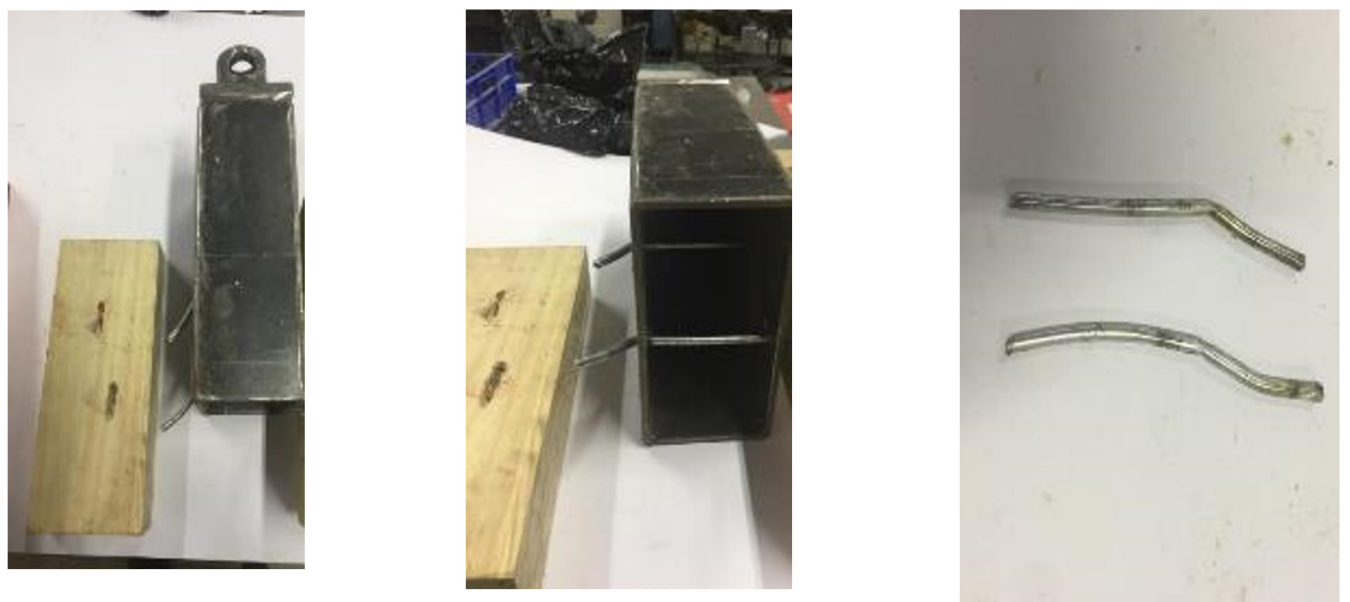

Figure 5. Ductile failure after fatigue of the dowels in the CLT-Steel connection. 


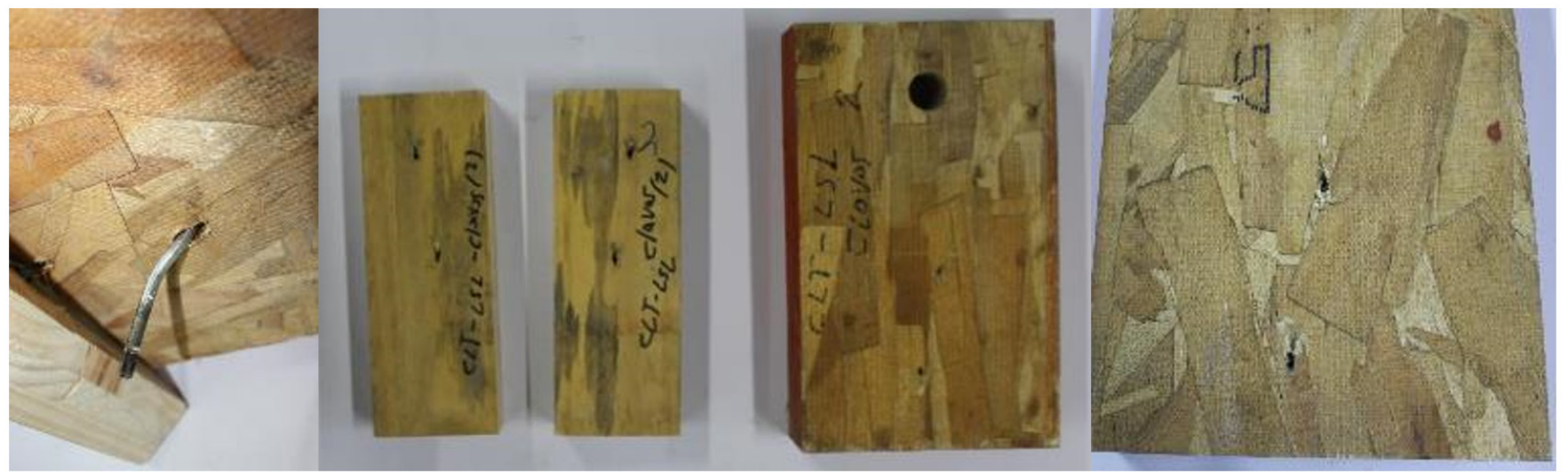

Figure 6. Ductile failure of the nails after fatigue in the CLT-LSLn connections.

The CLT-LSL joint allowed for assessing the effect of using different fasteners, as dowels, screws and nails were used as connectors. Not surprisingly the screwed connection showed the smallest ultimate displacement and ductility because the large withdrawal capacity do not allow for large flexural curvatures of the fastener prior to failure. Also, as expected, the nailed connection provided the largest ultimate displacements and ductility because, oppositely to screws, the small axial widrhawal capacity allowed for nailing axial extraction yielding to larger slips at the shear plane. The comparison of the distinct connectors' capacities is not deemed relevant because, as all the connections were designed to fail under ductile modes, the capacity directly relates with the yielding moment of the connector, which in turn is just defined by the yielding strength of the steel and the effective diameter of the connector. In terms of initial slip, as expected, the dowelled connection showed certain looseness caused by the clearance holes of the predrilling in comparison to the dowelled and nailed connections but still the stiffness of the dowelled connection was very high.
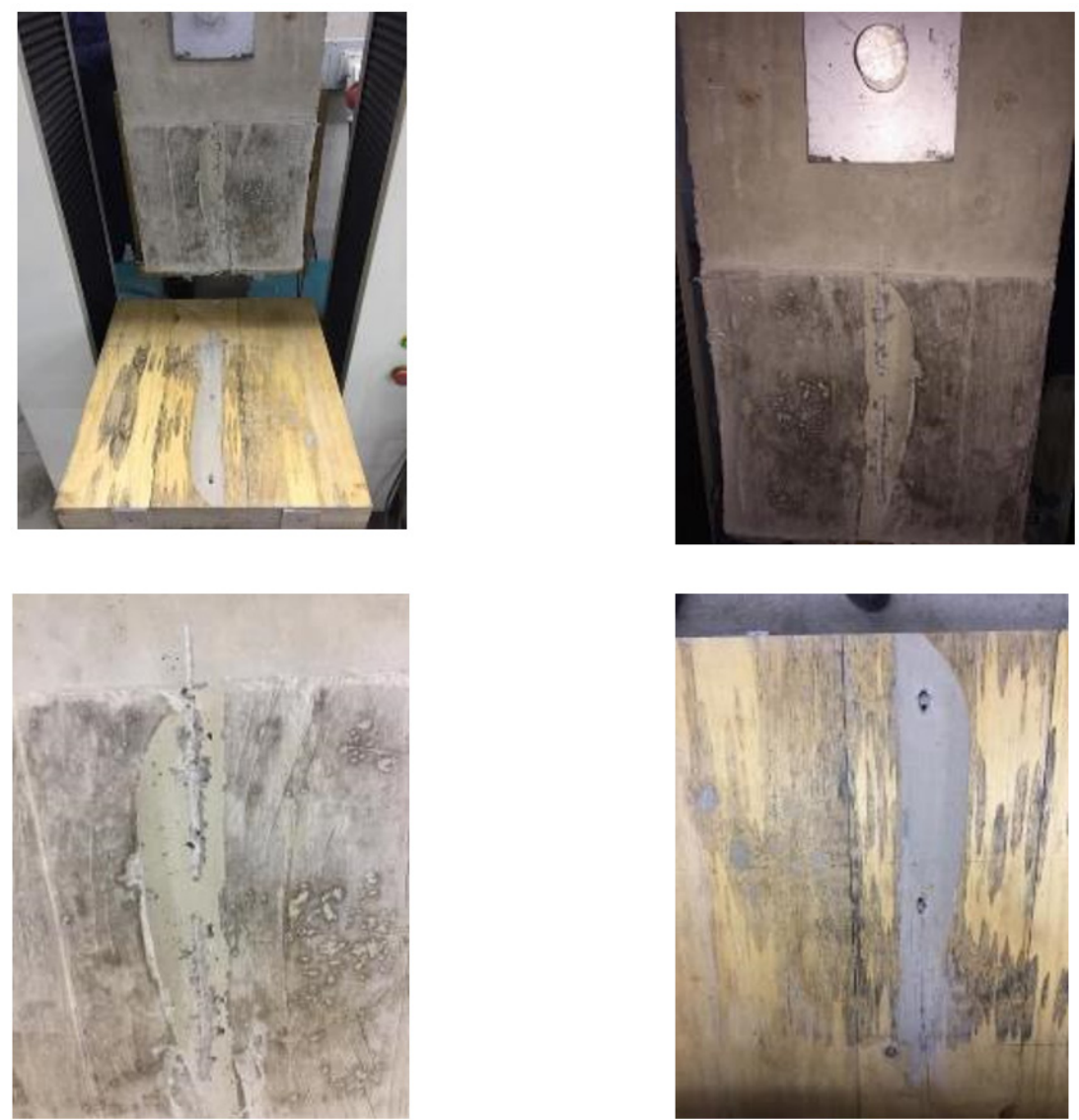

Figure 7. Brittle failure of the CLT-RCwo connection (without posttension bars). The failure was similar to the previous CLT-RCW but not so abrupt which is evidenced by less visible damage at the shear plane in the concrete. 


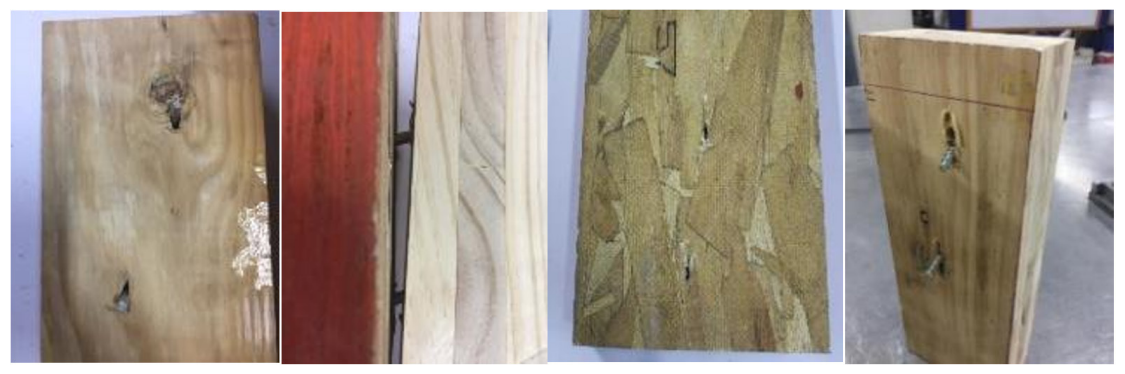

Figure 8. Brittle and premature failure of the self-tapping screws after fatigue at the CLT-LSLs connection.

The CLT-Steel connection allowed for an interesting comparison by using the same type of connector (steel self-drilling dowels) in distinct materials as these connectors are exactly the same as those used in the CLT-LSLd connection. The main observed difference between these two connections was the stiffness and ductility as both parameters were approximately twice larger in the CLT-Steel connections. The reason is that the hollowed steel profile served as a fixed end for the dowels increasing significantly the stiffness at the shear plane. This caused in turn a considerable reduction of about $50 \%$ of the yielding displacements in the CLT-Steel connection, which however showed almost the same ultimate displacements as the CLT-LSLd connetion thus yielding to a two-times-larger ductility for the steel connection. Actually, the ductility achieved with the CLT-Steel connection was even larger than the nailed CLT-LSL connection.

Table 1. Summary of the monotonic performance of the hybrid connections

\begin{tabular}{ccccc}
\hline Parameter & CLT-Steel & CLT-LSLs & CLT-LSLn & CLT-LSLd \\
\hline$K(\mathrm{kN} / \mathrm{mm})$ & 2.30 & 0.76 & 0.86 & 3.13 \\
$F_{y}(\mathrm{kN})$ & 5.3 & 7.6 & 3.0 & 4.2 \\
$\delta_{y}(\mathrm{~mm})$ & 2.98 & 10.0 & 3.48 & 6.4 \\
$F_{\max }(\mathrm{kN})$ & 8.20 & 9.0 & 6.22 & 7.32 \\
$\delta_{u}(\mathrm{~mm})$ & 26.5 & 21.5 & 30 & 26.3 \\
$D$ (unitsless) & 8.89 & 2.15 & 8.62 & 4.11 \\
\hline
\end{tabular}

\subsection{Cyclic performance}

A comparison of the hysteresis envelopes, energy dissipation (ED), equivalent viscous damping, strength degradation and stiffness degradation of the CLT-Steel, CLT-LSL and CLT-RC connections is shown in the Figure 9. Except for the CLT-RC specimens, in where only one test per design was conducted (one for CLT-RCW and one for CLT-RCWo), the curves of Figure 9 represent the average response of two cyclic tests. The full hysteretic curves of all the tests are detailed in the Figure 10. Similarly to Section 3.1, all the results of this section are corresponding to the response of one fastener and shear plane. 

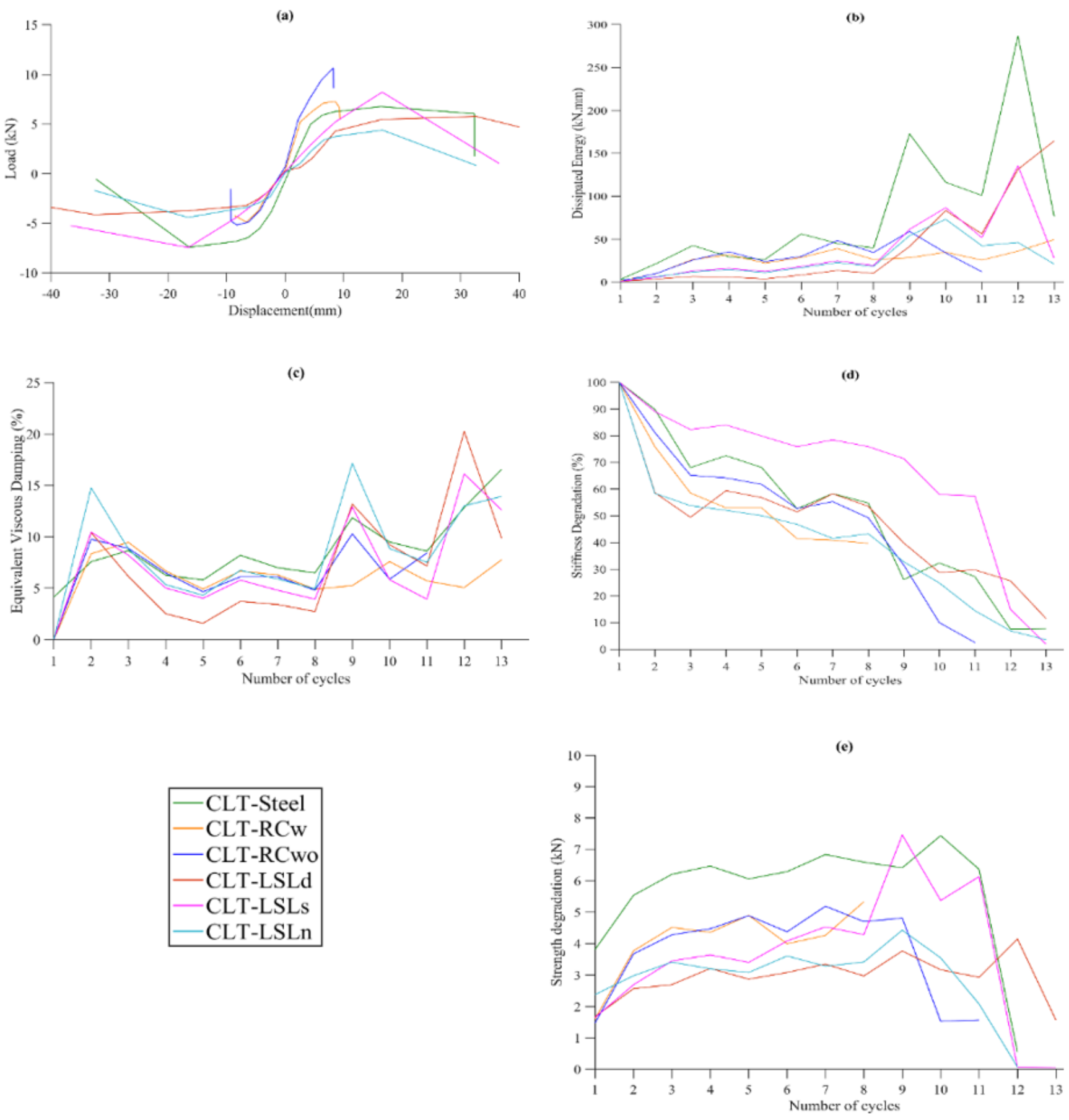

Figure 9. Comparison of the cyclic performance of the hybrid connections including; (a) hysteresis envelopes; (b) energy dissipation per cycle; (c) equivalent viscous damping per cycle; (d) stiffness degradation per cycle; (e) strength degradation per cycle.

The envelope curves shown in the Figure 9(a) were obtained as the average of the absolute values of the load and the displacement of the corresponding positive and negative envelope points for each cycle as stated in ASTM (2011). Out of the Figure 9(a), two very interesting observations can be made. The first is that all the connections exhibited a very high ductility under cyclic reverse loading - which is coherent with the monotonic response of Section 3.1, except the reinforced concrete connections in which ductility was significantly smaller. Actually, note that the screws used in the CLT-RC connections are the same as those of the CLT-LSLs connections, however the ductility of the later was over 3 times larger. The main reason for this decrease of ductility is that, under concrete, the screws experience virtually no any withdrawal dislplacement thus greatly increasing the stresses due to very large axial forces at the connectors. Such stress concentration is also accompanied by a shear stress concentration at the boundaries of the concrete, which 
triggers the brittle failure of the concrete at the shear plane resulting in the shear-off and so the brittle failure of the fasteners; see the full details of the distinct failure modes in Appendix A. The second interesting fact is that for all the connections the envelopes behaved relatively symetric for tension and compresion except for the reinforced concrete connections, in which a dramatic assymetry was evidenced. Such assymetry is attributed to a substantial increase of friction forcese at the shear plane (rope effect) which are greatly increased not only because the friction coefficient of wood-to-concrete is larger, but especially because the self tapping screws were axialy restricted in the concrete yielding to very large axial forces at the screws and so very large compression forces at the shear plane. This behaviour is assymetric because friction only occurs in one direction of loading. Unexpectedly, the capacity of the postensioned connection (CLT-RCW) was smaller than the un-postensioned connection (CLT-RCwo). Also, one may believe that the postensioned connection would be stiffer than the unpostensioned due to increased compression at the shear plane due to possion deformation but it was not the case. The reason that explains these phenomena is attributed to an abrupt and premature failure of the concrete at the shear plane for the CLT-RCW connections, i.e. the posttensioning stressess increased the local embedment stress near the connectors triggering premature abrupt failure modes of the concrete at the shear plane and thus the shear-off of the screws (see Appendix A). Except for the aforementioned two remarkable observations, the ductilities and stifnesses showed by the remaining connections (CLT-Steel and CLT-LSL) were as anticipated by the monotonic experiments (Section 3.1).
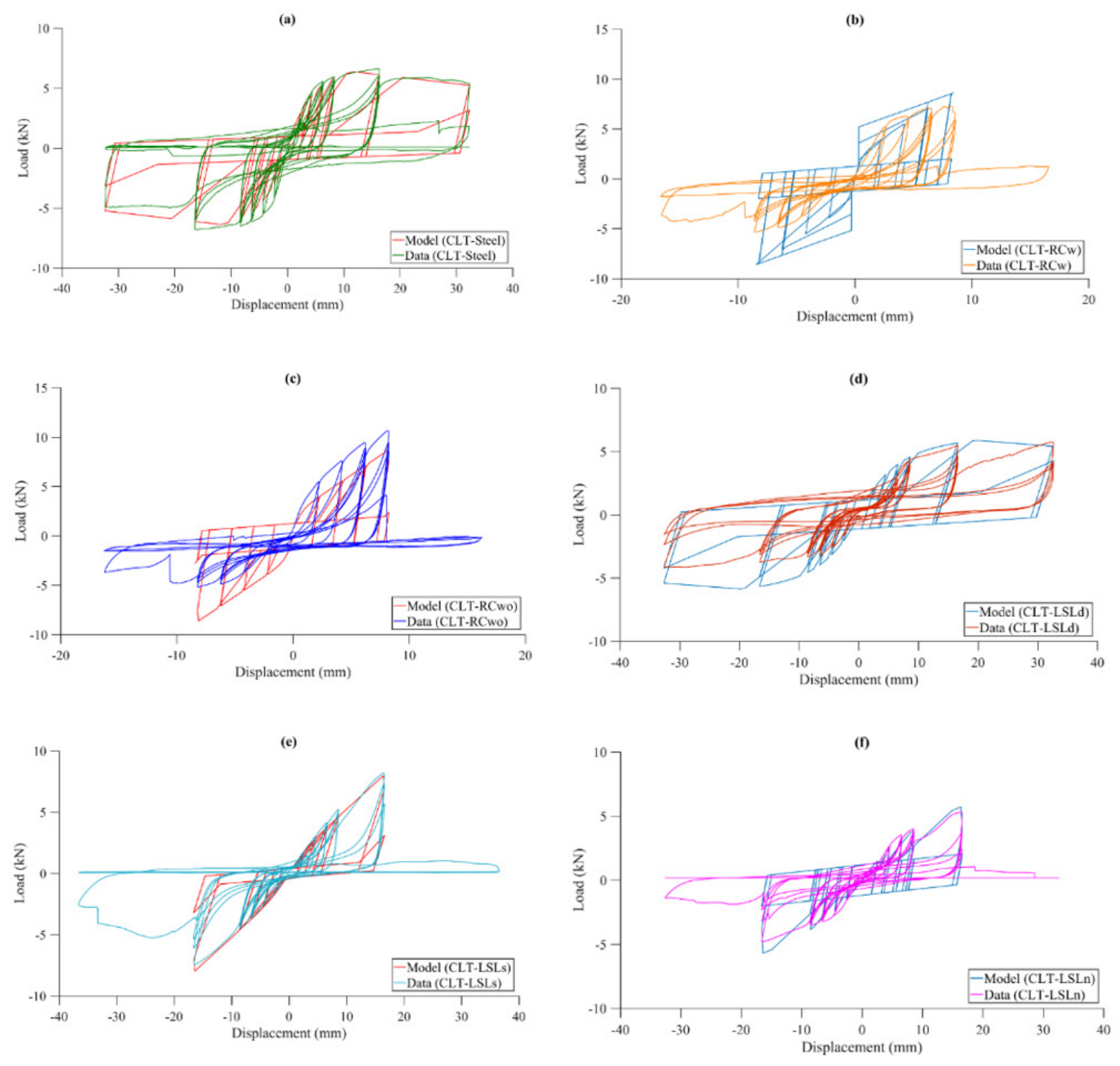

Figure 10. Full details of the cyclic hysteresis curves and corresponding numerical fit of each connection including: (a) CLT-Steel; (b) CLT-RCw; (b) CLT-RCwo; (d) CLT-LSLd; (e) CLT-LSLs; (f) CLT-LSLn. 
The results of the energy dissipation per cycle shown in Figure 9(b) evidence that at early stages (first cycles) of deformation, the CLT-RC and CLT-Steel connections dissipate about twice the energy of the remaining connections. This enhanced dissipation capability at early stages is mainly due to much stiffer connections in comparison to wood-to-wood connections. Also, it is interesting that the energy dissipation of the CLT-RC connections is relatively stable which indicates that these connections showed less pinching (narrowing of the hysteretic curves) due to less loosening of the fasteners at the shear plane. Another interesting fact is that the CLT-LSLs and CLT-LSLd, and especially the CLT-Steel connections, showed a very large energy dissipation at large deformation cycles due to high deformation of the connectors. Similar behavior is expected for the nailed CLT-LSL connections, but the lesser energy dissipation of these connections found in this investigation is attributed because the design of these connections was less resistant and stiff in comparison with the other fasteners. For all these connections however, the energy dissipation at high cycles is largely unstable due to very large pinching (see the full hysteresis curves in the Figure 10). Figure 10 shows full details of the cyclic hysteresis curves and corresponding numerical fit of each connection including: (a) CLT-Steel; (b) CLT-RC; (c) CLT-LSL.

The equivalent viscous damping, Figure 9(c), was relatively constant for all the tested connections. The most important damping is that showed in between the yield and ultimate deformations because it is the most representative to keep away brittle damage of structural wooden members during a seismic event. In that range of deformations, the Equivalent Viscous Damping (EVD) calculated as stated in Equation 1 was about 8,1\%, 7,3\% and 8,4\% for the CLT-Steel, CLT-RC and CLT-LSL connections.

The stiffness degradation presented in the Figure 9(d) shows that in overall all connections suffered severe stiffness degradation. Only the screwed CLT-LSL connection showed slightly slower degradation, but the stiffness degradation at large amplitude cycles was as higher as for all the connections. The stiffness degradation of the posttensioned RC connection seems lower, but this is because simply failed under premature loading, as the un-tensioned RC showed higher stiffness degradation. Finally, the strength degradation was also very significant for all the connections, but especially for the CLT-Steel connection, the degradation was very high as evidenced in the 'peaky' nature of the energy dissipation curve shown in Figure 9(b). This is attributed to the fact that for this connection all the damage is concentrated only in the side wooden members of the connection yielding to large capacity drops during load repetitions.

\section{NUMERICAL MODELLING}

Because the nature of the of the hysteresis curves of the tested hybrid connections was relatively similar to those of conventional wood-to-wood connections, the modelling of the connections was performed with the modified Stewart hysteretic model (MSTEW) proposed by Folz \& Filiatrault (2002), which has demonstrated very accurate results for modelling conventional timber connections in previous research, see e.g. Pang, W. et al. (2007) and Estrella, X. et al. (2020). The MSTEW model consists of 10 modeling parameters, which phenomenologically capture the crushing of the wood and yielding of the connectors occurring at the shear plane such that the nonlinear backbone envelope curve of the model is represented by the following set of equations (see a graphical explanation of each parameter in the Figure 11):

$$
\begin{aligned}
& F(\delta)= \\
& \operatorname{sgn}(\delta) \times\left(F_{0}+r_{1} K_{0}|\delta|\right) \times\left[1-\exp \left(-K_{0}|\delta| / F_{0}\right)\right] \\
& |\delta| \leq\left|\delta_{u}\right| \\
& F(\delta)= \\
& \operatorname{sgn}(\delta) \times F_{u}+r_{2} K_{0}\left[-\operatorname{sgn}(\delta) \times \delta_{u}\right] \\
& |\delta| \leq|\delta| \leq\left|\delta_{F}\right| \\
& F(\delta)=
\end{aligned}
$$

\section{0}

$|\delta|>\mid \delta_{F}$ 


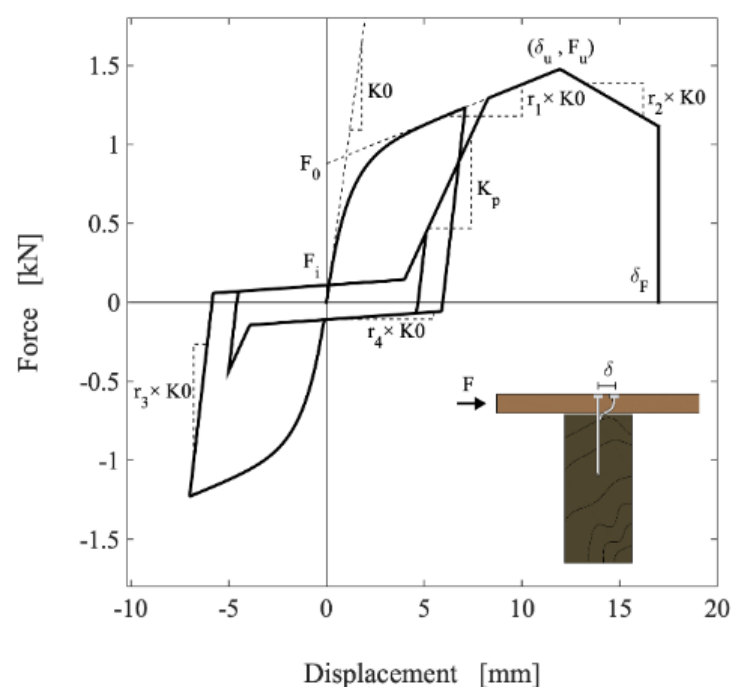

Figure 11. Illustration of the parameters used for modelling the hysteretic response of the modified Stewart model hysteretic model (MSTEW) proposed by Folz \& Filiatrault (2002), based on Estrella, X. et al. (2020).

As shown in the Figure 11, six stiffnesses are considered in the model during cyclic loading: (1) elastic stiffness, $\mathrm{K}_{0}$; (2) elasto-plastic stiffness with hardening during loading, $K_{0} r_{1} ;(3)$ elasto-plastic stiffness with softening at advanced deformations of loading, $K_{0} r_{2} ;(4)$ unloading off stiffness $r_{3} K_{0}$; (5) reloading stiffness $K_{p}$; and (6) continued unloading reduced stiffness $r_{4} K_{0}$. It is important to note that two different inelastic displacements $\delta_{u}$ (maximum displacement prior softening) and $\delta_{\mathrm{F}}$ (failure displacement) are assumed being both related with the 6 parameter. Two important forces are also assumed including $F_{0}$ (force given by the intersection of the ordinate axis with the secant of the elastoplastic stiffness) and $\mathrm{F}_{\mathrm{i}}$ (intersection between ordinate axis and reloading stiffness) being $\alpha$ a parameter that relates $\mathrm{K}_{\mathrm{p}}$ with $\mathrm{F}_{0}$ and $\mathrm{K}_{0}$, see further details in (Folz \& Filiatrault, 2002).

In this research, the above model was assumed for all the connections and the corresponding 10-parameter identification for each of the connections was obtained by nonlinear functional minimization procedures. More specifically, the parameters were calibrated in order to minimize the cumulative energy error, which expresses the summation of the areas within the hysteresis curve of each cycle and can be calculated as

$C E E=\frac{\left|E_{i t}-E_{i m}\right|}{\left|E_{i t}\right|}$

where $\mathrm{CEE}=$ cumulative energy error, and $\mathrm{E}_{\mathrm{it}}$ and $\mathrm{E}_{\mathrm{im}}$ are the total energy dissipated up to the ith time step of the test result and the model response, respectively. The resulting parameters along with corresponding fits of the cumulative energy error are presented in the Table 2, whereas a comparison between the experimental versus numerical cumulative energies is illustrated in the Figure 12. The full details of the comparison between experimental results and numerical simulations for each hysteresis curve is presented in the Figure 10.

Table 2. MSTEW hysteretic modelling parameters.

\begin{tabular}{|c|c|c|c|c|c|c|}
\hline Parameter & CLT-Steel & CLT-RCw & CLT-RCwo & CLT- LSLd & CLT-LSLS & CLT-LSLn \\
\hline$\kappa_{0}(\mathrm{kN} / \mathrm{mm})$ & 2.31 & 4.68 & 5.30 & 0.99 & 0.39 & 0.66 \\
\hline$r_{1}$ (unitsless) & 0.0033 & $1 e-09$ & 0.15 & 0.016 & 0.75 & 0.20 \\
\hline$r_{2}$ (unitsless) & -0.085 & -0.202 & -2.072 & -0.036 & -0.684 & -6.376 \\
\hline$r_{3}$ (unitsless) & 1.248 & 1.704 & 1.460 & 1.543 & 2.143 & 3.673 \\
\hline$r_{4}$ (unitsless) & 0.010 & 0.012 & 0.017 & 0.031 & 0.030 & 0.081 \\
\hline$F_{0}(\mathrm{kN})$ & 6.7 & 11.6 & 2.2 & 5.8 & 2.2 & 4 \\
\hline $\boldsymbol{F}_{1}(\mathrm{kN})$ & 1.1 & 1.8 & 1.2 & 1.1 & 0.3 & 1.2 \\
\hline$\delta_{u}(\mathrm{~mm})$ & 16.1 & 9.2 & 8.1 & 19.9 & 17.6 & 16.2 \\
\hline $\boldsymbol{\alpha}$ (unitsless) & 0.95 & 0.94 & 0.84 & 1 & 0.86 & 0.8 \\
\hline B (unitsless) & 1.25 & 1.24 & 1.36 & 1.84 & 1.30 & 1.1 \\
\hline CEE (error fit, \%) & -3.54 & 39.58 & 55.98 & 3.02 & 17.79 & 7.64 \\
\hline
\end{tabular}



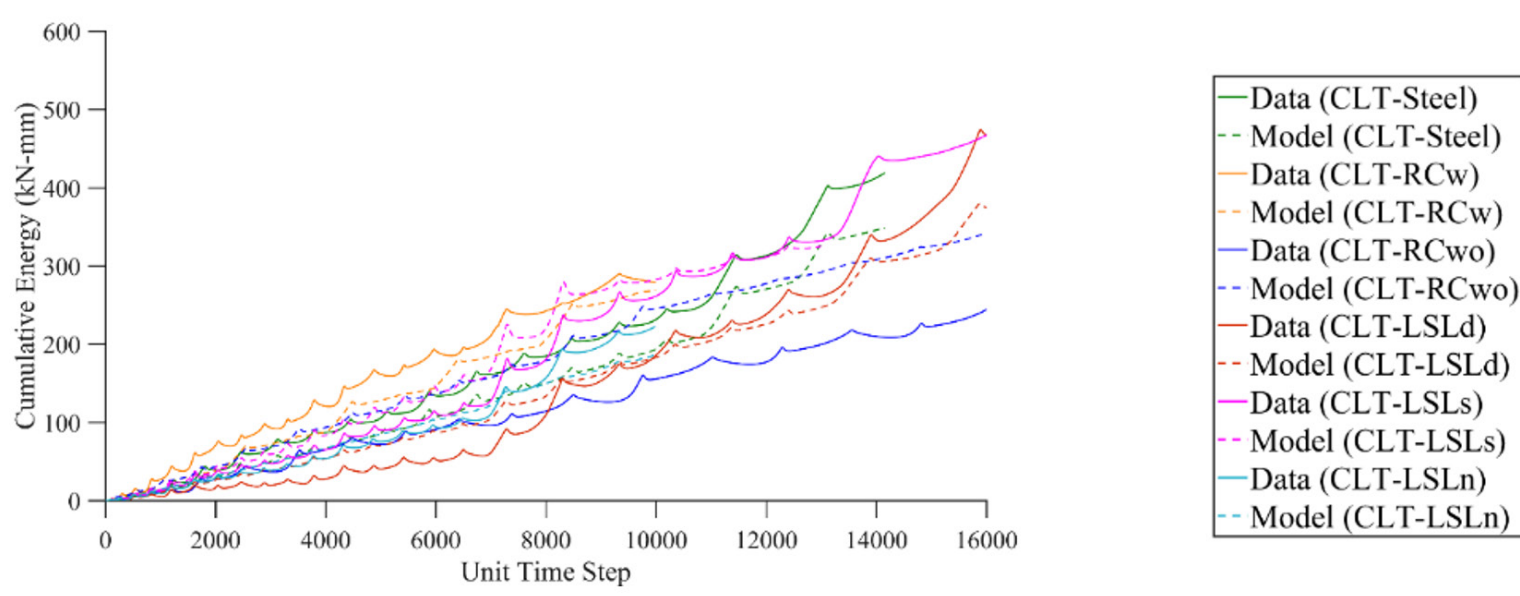

Figure 12. Comparison of the cyclic performance of the hybrid connections cumulative energy dissipation.

From the results of the numerical model, it can be concluded that the MSTEW hysteretic model is adequate for modelling these type of hybrid connections as the errors are below $10 \%$. However, larger errors of about 15 to $20 \%$ can be expected for connections with high axial forces at the connectors (rope effect) due to the asymmetry of the hysteresis curve such as in the case of using self-tapping screws. In these cases, the model always tends to overestimate the energy dissipation, stiffness, capacity and ductility of the compressive quadrant because the calibration of most parameters is performed according to the response of the first quadrant (tension). When using hybrid timber-reinforced concrete connections such errors can be indeed much larger up to $55 \%$ and above - even when the fit of the tensile response may seem almost perfect - due to a very large asymmetries caused by the rope effect.

\section{CONCLUSIONS}

This research accomplished the static and dynamic characterization and modelling of mechanical connections for hybrid materials' compositions that can be typically used in a range of mid and high-rise timber buildings, including combinations of cross-laminated timber (CLT) with posttensioned and un-tensioned reinforced concrete (RC), hollow structural steel profiles, and laminated strand lumber (LSL). More specifically, the research focused on direct jointing of distinct materials via mechanical fasteners, which has not been widely investigated in comparison to indirect connection technologies. The results indicated that these types of direct hybrid connections can be accurately designed using current design practices for common timber connections, achieving the anticipated mechanical capacities and failure modes, however, showing much larger inelastic displacements - of about $200 \%$ in comparison to conventional connections. In addition, for the CLT-Steel connections, the elastic stiffness can duplicate the values of common timber-to-timber connections using the same fasteners. These results suggest direct jointing of hybrid connections as an attractive alternative for on-site construction of composite buildings, but especially for prefabricated composite assemblies at the industry, because direct jointing is typically faster and cheaper than indirect jointing at prefabrication plants. Furthermore, most of the studied connections are recommended not only for bearing static but also dynamic loads due to high ductility along with high-energy dissipation capabilities and equivalent viscous damping. However, it is important to note that reparation work can be needed at extreme loading events such as major earthquakes because large stress and stiffness degradation was observed at large displacement amplitudes.

Although predictable and highly ductile behavior was found for most of the tested connections, brittle behavior was observed for the CLT-RC connections. The relevant failure modes observed in the distinct connections. Especially for the post-tensioned concrete connections, abrupt failure loading at premature loading stages can be expected due to brittle shear failure of the concrete at the shear plane. Therefore, hybrid RC connections are only recommended for connections not being subjected to large dynamic loads such as earthquake or wind loading. Another important aspect of hybrid RC connection is that the dynamic response is highly dependent upon the load direction, which can be evidenced by the asymmetry of the hysteretic response. The asymmetry is produced mainly because high friction at the shear plane occurs only in the first direction of loading.

Existing hysteresis models such as the MSTEW model can be used to accurately predict the monotonic as well as cyclic response of hybrid connections. Errors below $10 \%$ can be expected in general when reproducing the hysteresis curve. However, larger errors can be expected for connections subjected to high axial loading of the fasteners because 
of the asymmetry produced by the frictional effects. Such inaccuracy due to asymmetry turned most evident for the RC connections, for which the errors may be above $50 \%$. This makes evident that future research is needed for enabling existing hysteresis models to capture the asymmetric hysteretic behavior, which should be feasible but requires definition of further hysteretic parameters.

\section{Acknowledgements:}

This research has been funded by CONICYT Chile, FONDECYT PROJECT 11170863 and the APC was also funded by FONDECYT PROJECT 11170863. The authors thank Conicyt for the financial support, as well as the structural lab team of the Laboratory of Structural Engineering of the UC and the team of the Timber Innovation Center CIM-UC.

Author Contributions: Conceptualization, P Guindos, J Montaño and H Santa María; Methodology, T Carrero; Validation, T Carrero; Formal analysis, T Carrero and P Guindos; Investigation, T Carrero; Resources, P Guindos; Writing-original draft, P Guindos and T Carrero; Writing-review \& editing, T Carrero, P Guindos and H Santa María; Supervision, P Guindos and H Santa María; Project administration, P Guindos; Funding acquisition, P Guindos. All authors have read and agreed to the published version of the manuscript.

Editor: Marcílio Alves.

\section{References}

Aburto, V., (2014). Diseño de Losas de Madera Contralaminada [Design of Contralaminated Wood Slabs], Civil and Environmental Engineer, Universidad del Bio-Bio, Chile, July.

Aicher, S.; Reinhardt, H.-W.; \& Garrecht, H., (2014). Materials and Joints in Timber Structures Recent Developments of Technology. Springer: New York London; Vol. 9, pp. 157-307. Retrieved from http://www.springer.com/series/8781

ASTM Standard E 2126, (2011). Standard Test Methods for Cyclic (Reversed) Load Test for Shear Resistance of Vertical Elements of the Lateral Force Resisting Systems for Buildings. West Conshohocken, PA, USA.

Blaß, H. J.; \& Sandhaas, C., (2017). Timber Engineering Principles for Design. In Timber Engineering, 1st ed.; Scientific Publishing: Karlsruhe, Germany; pp. 249-263.

Chapman, J.; Reynolds, T.; \& Harris, R. (2012). A 30 Level Cross Laminated Timber Building System and Analysis of the Eurocode Dynamic Winds Loads. In World Conference on Timber Engineering, New Zealand, 15-19 July.

Connolly, T., Loss, C., Iqbal, A., \& Tannert, T., (2018). Feasibility Study of Mass-Timber Cores for the UBC Tall Wood Building. World Conference on Timber Engineering, Republic of Korea, August 20-23, 2018. DOI:10.3390/buildings8080098

Dickof, C., (2013). CLT Infill Panels in Steel Moment Resisting Frames as a Hybrid Seismic Force Resisting System, Master of Applied Science, The University of British Columbia, Canada, April.

Dunbar A; Moroder D; Pampanin S; \& Buchanan A., (2014). Timber Core-walls for Lateral Load Resistance of Multi-storey Timber Buildings. New Zealand Timber Design Journal, Vol. 22, pp 11-19.

Estrella, X.; Guindos, P.; Almazán, J.L.; \& Malek, S., (2020). Efficient nonlinear modeling of strong wood frame shear walls for mid-rise buildings. Engineering Structures 2020, Vol. 215, pp. 110670. https://doi.org/10.1016/j.engstruct.2020.110670.

European Cooperation in Science \& Technology, (2018). Design of Connections in Timber Structures: A state-of-the-art report by COST Action FP1402 / WG3 Editors: Sandhaas, C., Munch-Andersen, J., Dietsch, P., Publisher: Shaker Verlag Aachen, Germany, pp.241-295.

European Committee for Standardization, (2005). DIN EN 12512. Holzbauwerke - Prüfverfahren - Zyklische Prüfungen von Anschlüssen mit mechanischen Verbindungsmitteln [Timber Structures Test Methods Cyclic Testing of Mechanical Connections].

European Committee for Standardization, (2009). Eurocode 8: Design of Structures for Earthquake Resistance - Part 1: General rules, seismic actions and rules for buildings, European Committee for Standardization: Brussels, July. Retrieved from https://www.phd.eng.br/wp-content/uploads/2015/02/en.1998.1.2004.pdf 
European Committee for Standardization, (2016a). EN 338: Structural Timber - Strength classes. Bruxelles, Belgium.

European Committee for Standardization, (2016b). EN 1995-1-1: Eurocode 5: Design of Timber Structures - Part 1-1: General Common rules and rules for buildings. Brussels.

Federal Emergency Management Agency, (2000). FEMA 356 Prestandard and Commentary for the Seismic Rehabilitation of Buildings, 1st ed; Ingle, K., American Society of Civil Engineers: 1801 Alexander Bell Drive, Reston, Virginia, 20191, United States of America.

Federal Emergency Management Agency, (2009). FEMA P695 Quantification of Building Seismic Performance Factors Washington, DC.

Folz, B.; \& Filiatrault, A., (2002). A Computer Program for Cyclic Analysis of Shearwalls in Woodframe Structures. Consortium of Universities for Research in Earthquake Engineering, Richmond, CA 94804, United States of America.

Foster, R. M.; Reynolds, T. P.; \& Ramage, M. H., (2016). Proposal for Defining a Tall Timber Building. Journal of Structural Engineering, Vol. 142(12), 02516001.

General Building Inspectorate Approval, (2014). DIBt 153-1.9.1-501/10 Deutsches Institut Für Bautechnik [German Institute of Civil Engineering]. Berlin.

Guíñez, F.; Santa María, H.; \& Almazán, J. L., (2019). Monotonic and Cyclic Behaviour of Wood Frame Shear Walls for MidHeight Timber Buildings. Engineering Structures, Vol. 189, pp. 100-110. DOI:10.1016/j.engstruct.2019.03.043

Hashemi, A.; Masoudnia, R.; \& Quenneville, P., (2016). Seismic Performance of Hybrid Self-Centring Steel-Timber Rocking Core Walls with Slip Friction Connections. Journal of Constructional Steel Research, Vol. 126, pp. 201-213.

DOI:10.1016/j.jcsr.2016.07.022

Hashemi, A.; Masoudnia, R.; Zarnani, P.; \& Quenneville, P., (2018). Seismic Resilient Cross Laminated Timber (CLT) Platform Structures Using Resilient Slip Friction Joints (RSFJs). In World Conference on Timber Engineering. Seoul, Republic of Korea, August 20-23.

Hassanieh, A.; Valipour, H. R.; \& Bradford, M. A., (2016). Load-Slip Behaviour of Steel-Cross Laminated Timber (CLT) Composite Connections. Journal of Constructional Steel Research, Vol. 122, pp. 110-121. DOI:10.1016/j.jcsr.2016.03.008

Hummel, J., (2017). Displacement-Based Seismic Design for Multi-Storey Cross Laminated Timber Buildings, Doctor in Engineering, Kassel University, Germany.

Iqbal, A.; Pampanin, S.; Palermo, A.; \& Buchanan, A. H., (2015). Performance and Design of LVL Walls Coupled with UFP Dissipaters. Journal of Earthquake Engineering, Vol. 19(3), pp. 383-409. DOI:10.1080/13632469.2014.987406

Kanazawa, K.; Kitamori, A.; Yamanishi, T.; Nakashima, S.; Araki, Y.; \& Isoda, H., (2018). A Case Study On In-Plain Shear Performance Of Composite Structure With CLT Infilled In Steel Frames. In World Conference On Timber Engineering. Seoul, Republic of Korea, August 20-23.

Koshihara, M.; Isoda, H.; \& Yusa, S., (2013). The Design and Installation of a Five-Story New Timber Building in Japan, In Internationales Holzbau-Forum.

Loss, C.; Piazza, M.; \& Zandonini, R., (2016). Connections for Steel-Timber Hybrid Prefabricated Buildings. Part II: Innovative Modular Structures. Construction and Building Materials, Vol. 122, pp. 796-808. DOI:10.1016/j.conbuildmat.2015.12.001

LP Building Products, (2019). LP SolidStart Laminated Strand Lumber and Laminated Veneer Lumber PR-L280. The Engineering Wood Association: Louisiana-Pacific Corporation, July 21. Retrieved from https://Ipcorp.com/resources/product-literature/

Moore, M. (2000). Scotia Place-12 Story Apartment Building A Case Study of High-Rise Construction Using Wood and Steel. World Conference On Timber Engineering. Auckland, New Zealand.

Pang, W.; Rosowsky, D.; Pei, S.; \& Van De Lindt, J. W., (2007). Evolutionary Parameter Hysteretic Model for Wood Shear Walls. Journal Structural Engineering, Vol. 133(8), pp. 1118-1129. https://doi.org/10.1061/ASCE0733-94452007133:81118.

Ringhofer, A.; Brandner, R.; \& Blaß, H. J., (2018). Cross Laminated Timber (CLT): Design Approaches for Dowel-Type Fasteners and Connections. Engineering Structures, Vol. 171, pp. 849-861. DOI:10.1016/j.engstruct.2018.05.032

Schneider, J; Karacabeyli, E; Popovski, M; Stiemer, S F; \& Tesfamariam, S., (2014). Damage Assessment of Connections Used in Cross-Laminated Timber Subject to Cyclic Loads, Journal Perform. Constr. Facil., Vol. 28, pp. 1-14. DOI: 10.1061/(ASCE)CF.1943-5509.0000528. 
Van De Kuilen, J. W. G.; Ceccotti, A.; Xia, Z.; \& He, M., (2011). Very Tall Wooden Buildings with Cross Laminated Timber. Procedia Engineering, Vol. 14, pp. 1621-1628. DOI:10.1016/j.proeng.2011.07.204

Wu, K.-Y.; Iqbal, A.; \& Popovski, M., (2018). Experimental Study Of Post-Tensioned CLT Wall Systems. World Conference On Timber Engineering. Seoul, Republic of Korea, August 20-23. 


\section{Appendix A}

In this section, full details of the hybrid connections' designs are presented, including: Figure 13, full detailling of the CLT-Steel connection's (CLT-Steel) design; Figure 14, full detailing of the CLT-LSL connection's design with self-tapping screws (CLT-LSLS). The components' dimensions and fasteners' spacing of the CLT-LSL joint with nails (CLT-LSLn) and dowels (CLT-LSLd) are the same as the CLT-LSLs; Figure 15, full detailing of the posttensioned CLT-RC connection with self-tapping screws (CLT-RCW). The details of the non-posttensioned CLT-RC joint with self-tapping screws (CLT-RCwo) are the same as the CLT-RCW except that PVC tubes, posttension bars and reaction plates are not used.

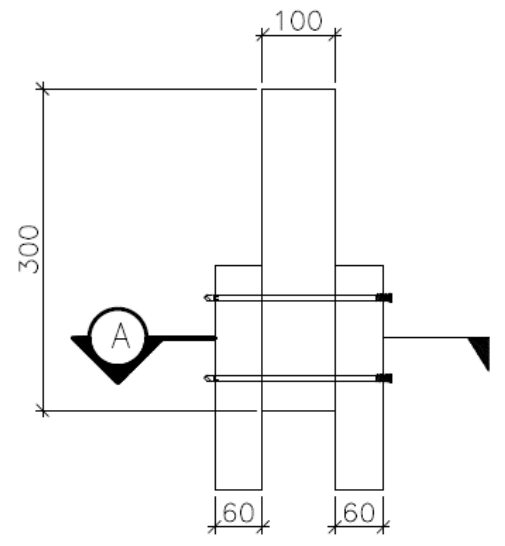

$\frac{\text { CLT-STEEL }}{\text { ESC }}$

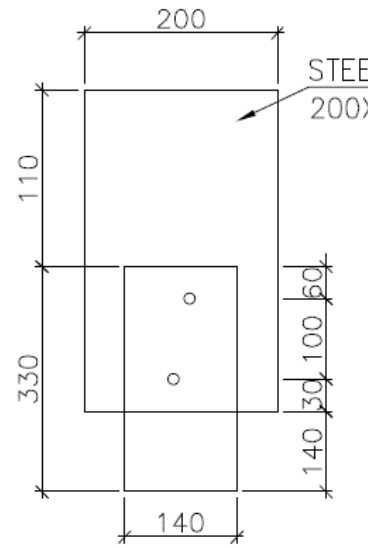

$200 \times 100 \times 6 \mathrm{~mm}$

Figure 13. Full detailing of the CLT-Steel connection's (CLT-Steel) design.

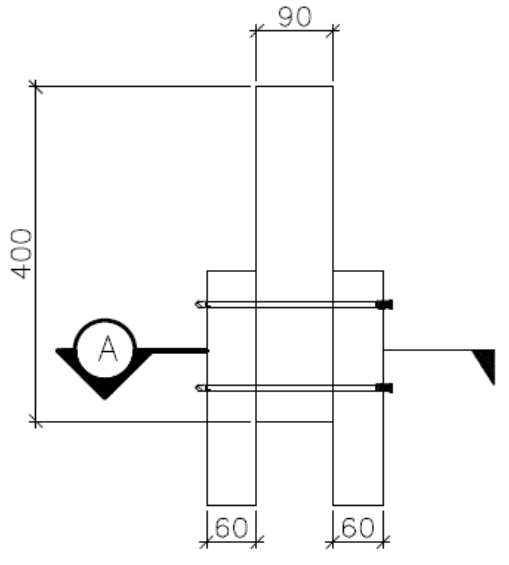

CLT-LSL
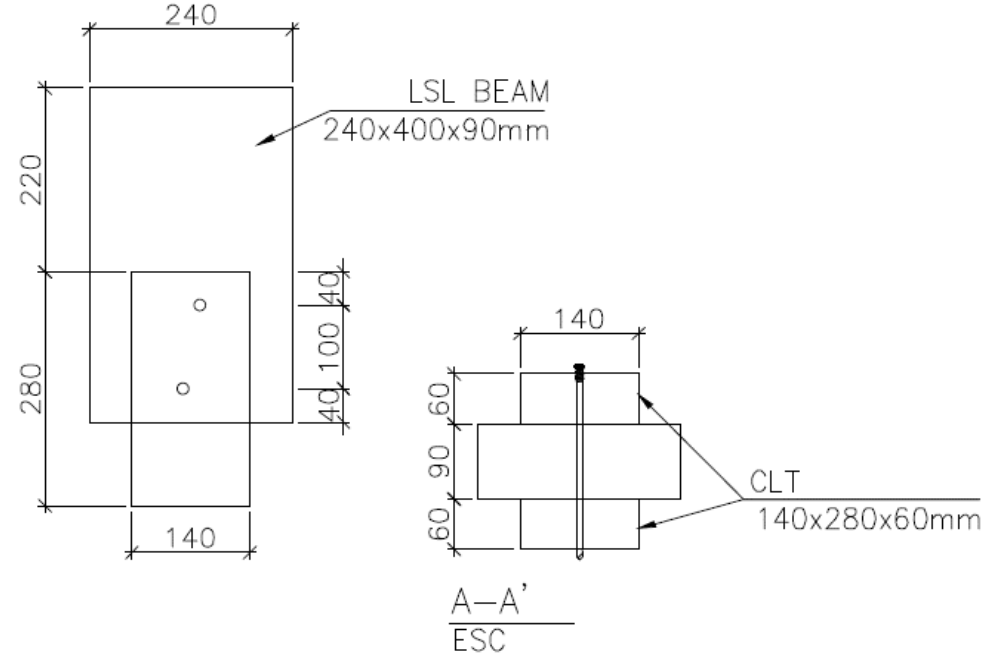

Figure 14. Full detailing of the CLT-LSL connection's design with self-tapping screws (CLT-LSLS). The components' dimensions and fasteners' spacing of the CLT-LSL joint with nails (CLT-LSLn) and dowels (CLT-LSLd) are the same as the CLT-LSLs. 


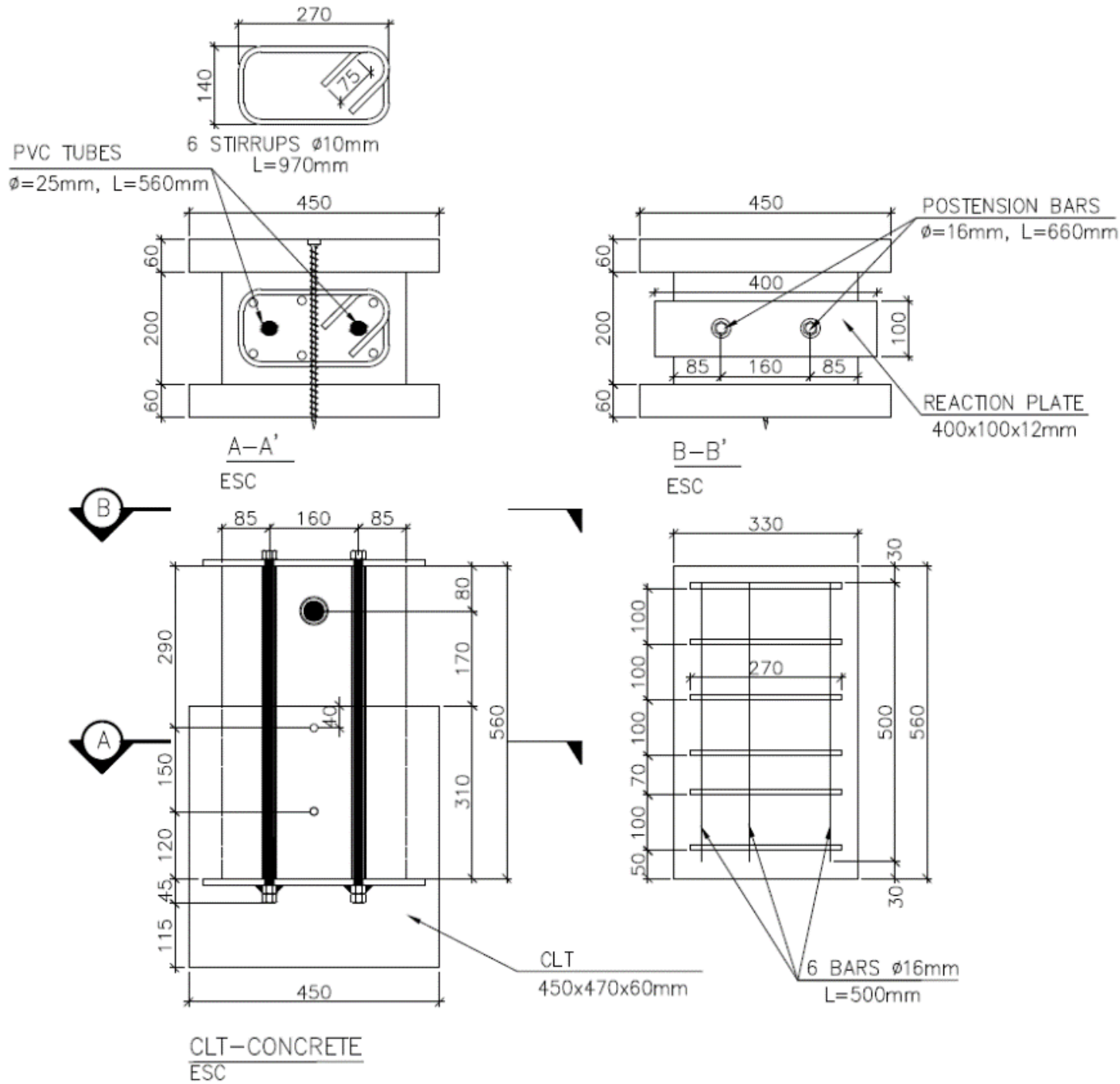

Figure 15. Full detailing of the posttensioned CLT-RC connection with self-tapping screws (CLT-RCW). The details of the non-posttensioned CLT-RC joint with self-tapping screws (CLT-RCWo) are the same as the CLT-RCW except that PVC tubes, posttension bars and reaction plates are not used. 